\title{
Improving Emotion Regulation with CBT in Young Children with High Functioning Autism Spectrum Disorders: A Pilot Study
}

\author{
Angela Scarpa and Nuri M. Reyes \\ Virginia Polytechnic Institute and State University, Blacksburg, USA
}

\begin{abstract}
Background and Aims: This pilot study tested the efficacy of a developmentally modified CBT for young children with Autism Spectrum Disorders (ASD) to teach emotion regulation strategies for reducing anger and anxiety, commonly noted problems in this population. Method: Eleven 5-7 year-old children participated in a CBT-group while parents participated in psychoeducation. Children were randomly assigned to an experimental or delayedtreatment control group. Results: From pre- to post-treatment, all children had less parent reported negativity/lability, better parent reported emotion regulation, and shorter outbursts, and also generated more coping strategies in response to vignettes. Parents also reported increases in their own confidence and their child's ability to deal with anger and anxiety. Conclusions: This study suggests that young children with high functioning ASD may benefit from CBT to improve regulation of anger and anxiety, and parent training may improve parental self-efficacy. Future studies are needed to make conclusions about its efficacy.
\end{abstract}

Keywords: Emotion regulation, anger and anxiety, children, autism.

\section{Introduction}

Current estimates suggest that as many as 1 in 110 children are diagnosed with an autism spectrum disorder (ASD), which includes Autistic Disorder, Asperger Syndrome, and Pervasive Developmental Disorder - Not Otherwise Specified (PDD-NOS). A hallmark of ASD involves difficulties with understanding their own and others' mental states. These deficits could affect the way children with ASD understand, interpret, and deal with their emotions and the emotions of others, which in turn may impair their ability to regulate their emotional experiences.

Although little is known about emotion regulation in children with ASD, children with ASD show increased rates of co-morbid anxiety (29-84\%) and disruptive behavior (28$62 \%$ ) disorders, for example, suggesting difficulties managing stress and anger (de Bruin, Ferdinand, Meester, de Nijs and Verheij, 2007). These deficits could, in turn, interfere with the ability to develop and maintain successful social interactions. As such, it is important to

Reprint requests to Angela Scarpa, Virginia Tech - Psychology, 3110 Prices Fork Road, Blacksburg, Virginia 24061, USA. E-mail: ascarpa@vt.edu. An extended version is also available online in the table of contents for this issue: http://journals.cambridge.org/jid_BCP 
help children with ASD understand and manage their emotions, and to provide them with this help as early as possible.

Few empirically-tested psychosocial interventions have been developed to improve emotion regulation in children with ASD. CBT has shown some success in addressing related emotional difficulties (Attwood, 2004). In particular, four studies to date have targeted anxiety (Chalfant, Rapee and Carroll, 2007; Sofronoff, Attwood and Hinton, 2005; Wood et al., 2009) and anger (Sofronoff, Attwood, Hinton and Levin, 2007) using randomized controlled designs (RCTs), thus providing evidence of the use of CBT for treating emotional dysfunction in this population.

Although these studies indicate that CBT can be useful for children with high-functioning ASD in terms of decreasing their anxiety/anger, previous treatment trials focused only on older children (i.e. children in late childhood and early adolescence). To the authors' knowledge, studies of CBT for emotion regulation in children with ASD younger than age 7 years have yet to be conducted. Since early intervention appears to be a predictor for a favorable prognosis in the quality of life of children with ASD, it would be important to begin teaching these skills as early as possible.

\section{Present study}

In the present study, the group CBT used by Sofronoff et al. $(2005,2007)$ was adapted to be developmentally appropriate for 5 to 7 year-old children with ASD. Developmental modifications included shorter sessions, greater use of songs, stories, and play activities, and the incorporation of parent-training. In addition to the child group session, a simultaneous psycho-educational parent group was included in which parents met with another therapist and were able to watch their children's sessions. The principal objective in this study was to investigate the efficacy of this CBT to teach emotional states and emotion regulation strategies to young children (aged 5-7) with high functioning ASD. Thus, this study investigated the following hypotheses: (1) CBT will benefit young children with high functioning ASD by improving their knowledge of emotion regulation strategies and their ability to regulate emotions, specifically related to anger and anxiety; (2) CBT will benefit parents through increases in parental self-confidence in their ability, as well as confidence in their child's ability, to manage the child's anger/anxiety.

\section{Method}

\section{Participants}

Participants included 11 European American children (two girls, nine boys), ranging from 4.5-7 years old at intake, and their mothers. Median household income was high $(\$ 85,000$; ranging from $\$ 14,400$ to $\$ 175,000$ ) compared to the median household income for Virginia in 2007-2008 (\$61,710). Children had to meet ASD criteria on the Autism Diagnostic Observation Schedule. They also had to be 5-7 years-old at the time of intervention, in kindergarten or first grade, verbal, and able to understand and follow verbal instructions. Full details on the study sample, design, measures, and results can be found in the extended online version. 


\section{Measures}

Child's emotion regulation ability was assessed through his/her report of emotion regulation strategies that could be used when dealing with anger and anxiety related emotions presented in vignettes (number of different strategies was summed), as well as parental report on an emotion regulation scale, and parental observations of frequency per hour and duration in minutes of anxiety/anger episodes. A scale was created for this study to assess parental selfconfidence and parental confidence in their children's abilities by asking two questions in which parents rated the extent to which they felt their children were able to handle their emotions related to anger or anxiety on a scale of 1 to 10 .

\section{Procedures}

After determining eligibility, children were randomly assigned to either the experimental $(n=5)$ or delayed-treatment control $(n=6)$ group, with 2-3 children per group. The delayed-treatment control group started the intervention approximately one week after the experimental group completed the intervention. The experimental group completed the outcome measures at Time 1 (intake) and Time 2 (post-treatment) whereas the delayedtreatment control group completed the same outcome measures at Time 1 (intake), Time 2 (pre-treatment), and Time 3 (post-treatment). One-tailed parametric and nonparametric comparisons were conducted, as appropriate.

\section{Intervention}

Child group sessions. Children attended one-hour group meetings for 9 consecutive weeks. All therapists, three clinical graduate students and two trained staff members, were trained and supervised by a licensed clinical psychologist. The intervention's treatment manual was followed and sessions were reviewed for treatment adherence. The treatment manual is available from the first author by request. The intervention focused on skill-building via affective education, stress management, and understanding expressions of emotions. A group therapy approach was used to teach the children to recognize emotions in themselves and others. The therapy facilitated emotion regulation by teaching relaxation, physical, social, and cognitive tools to "fix" intense emotions. Sessions were structured in such a way as to have a Welcome Time, Singing, Story Time, Activity/Lesson Time, Snack, and Goodbyes, all revolving around a particular topic. The topics for the nine sessions were:

Session 1: Understanding positive emotions of happiness.

Session 2: Understanding positive emotions of relaxation and negative emotions of anger and anxiety.

Session 3: Emotional Toolbox 1: Introduction and Teaching physical and relaxation tools.

Session 4: Emotional Toolbox 2: Teaching social tools.

Session 5: Emotional Toolbox 3: Teaching thinking tools.

Session 6: Emotional Toolbox 4: Teaching special interest tools.

Session 7: Emotional Toolbox 5: Identifying appropriate and inappropriate tools.

Session 8: Review Session: Create a production (similar to a commercial) to highlight tools to remember.

Session 9: Wrap-up: Farewell. 
Parent group meetings. Nine psychoeducational parent group sessions occurred simultaneously with the children's sessions. Parents met with another therapist to review and discuss session material and discuss/troubleshoot how strategies were being implemented outside of the clinic setting. They were also able to watch the children's sessions on a monitor to observe how lessons were being taught. Parents were provided with a handout outlining the session for every meeting and were given homework assignments to practice skills with their child.

\section{Results}

Comparisons at Time 2 of the experimental group (immediately after treatment) and delayedtreatment control group (prior to treatment)

A summary of findings is presented in Table 1. Results indicated significantly fewer outbursts as monitored by parents in the experimental group, and a trend toward shorter duration per episode in the experimental group, compared to the delayed-treatment control group. Children in the experimental group also responded with a significantly greater average number of strategies than the delayed-treatment control group in response to vignettes. Moreover, parents in the experimental group reported significantly higher levels of confidence in their ability to manage their child's anxiety, but not anger, than the delayed-treatment control group. Parents also reported significantly higher levels of confidence in their child's ability to deal with anger and anxiety than in the delayed-treatment control group.

\section{Comparisons of the whole sample before and after treatment}

Children showed significant reductions in the Negativity/Lability subscale and a trend towards increased scores on the Emotion Regulation subscale after treatment, suggesting lower intensity and better regulation of mood overall as reported by parents on an emotion regulation questionnaire. Parents also observed a trend for shorter duration per episode after treatment. Children responded to the vignettes with significantly more strategies to control anger or anxiety. Finally, parents reported significantly higher levels of confidence in their own and their child's abilities to deal with anger and anxiety-related emotions after treatment.

\section{Discussion}

Taken together, these findings suggest improved emotion regulation in that outbursts became fewer and shorter, mood intensity scores decreased, and mood regulation scores increased after treatment. Further, the treatment may increase children's knowledge of emotion regulation strategies as well as parental confidence in being able to manage their child's emotions. The findings are limited by the small sample size and initial group differences; thus they need to be replicated with larger samples and more sophisticated measures and analyses to address possible sample biases. Nonetheless, until recently, children with ASD have been treated with pharmacotherapy for anxiety and anger related difficulties, and new empirically based psychosocial interventions are needed. Although a handful of prior studies showed improvement in emotional functioning after CBT in older children (i.e. 8-14 years old), the current findings show similar patterns and suggest that a cognitive behavioral approach 
Table 1. Mean differences at Time 2 between the experimental group (immediately after treatment) and the delayed-treatment control group (prior to treatment)

\begin{tabular}{|c|c|c|c|c|}
\hline & $\begin{array}{l}\text { Experimental } \\
\text { group } \\
\text { Mean }(S D)\end{array}$ & $\begin{array}{l}\text { Delayed-treatment } \\
\text { control group } \\
\text { Mean }(S D)\end{array}$ & $\begin{array}{l}t \text {-values } \\
\text { or Mann } \\
\text { Whitney } \\
\text { U values }\end{array}$ & $\begin{array}{c}\text { Effect } \\
\text { size } \\
(d)\end{array}$ \\
\hline \multicolumn{5}{|l|}{ Emotion Regulation Checklist } \\
\hline Emotion Regulation Subscale & $23.20(2.68)$ & $23.43(1.99)$ & .17 & .05 \\
\hline Negativity/Lability Subscale & $35.80(4.71)$ & $34.57(3.91)$ & .49 & .15 \\
\hline \multicolumn{5}{|l|}{ Behavioral Monitoring Sheet } \\
\hline Frequency of episodes per hour & $\begin{array}{c}.16(.05) \\
\text { Mean rank } 3.80\end{array}$ & $\begin{array}{c}.17(.12) \\
\text { Mean rank } 7.00\end{array}$ & $4.00^{*}$ & .05 \\
\hline Duration in minutes per episode & $2.57(2.10)$ & $7.74(7.18)$ & $1.54+$ & .46 \\
\hline \multicolumn{5}{|l|}{$\begin{array}{l}\text { Ben and the Bullies and James } \\
\text { and the Reading Group Vignettes }\end{array}$} \\
\hline Quantity scores & $4(2.45)$ & $1.29(.95)$ & $2.70^{*}$ & .65 \\
\hline \multicolumn{5}{|l|}{ Self Confidence Rating Scale } \\
\hline Parental self-confidence - anger & $\begin{array}{l}7.75(1.26) \\
\text { Mean rank } 7.90\end{array}$ & $\begin{array}{l}5.43(1.62) \\
\text { Mean rank } 5.50\end{array}$ & 10.50 & .63 \\
\hline Parental self-confidence - anxiety & $\begin{array}{l}8(.71) \\
\text { Mean rank } 10.00\end{array}$ & $\begin{array}{l}4.14(1.68) \\
\text { Mean rank } 4.00\end{array}$ & $0.00^{*}$ & .84 \\
\hline Confidence in child - anger & $\begin{array}{l}6.80(.84) \\
\text { Mean rank } 10.00\end{array}$ & $\begin{array}{l}3.43(.98) \\
\text { Mean rank } 4.00\end{array}$ & $0.00^{*}$ & .89 \\
\hline Confidence in child - anxiety & $\begin{array}{l}6.60(.89) \\
\text { Mean rank } 10.00\end{array}$ & $\begin{array}{c}2.71(1.50) \\
\text { Mean rank } 4.00\end{array}$ & $0.00^{*}$ & .85 \\
\hline \multicolumn{5}{|c|}{$\begin{array}{c}{ }^{*} p<.05,+p<.10, \text { one-tailed independent t-test or Mann Whitney } \mathrm{U} \text { test. Mean ranks added } \\
\text { for Mann Whitney U tests }\end{array}$} \\
\hline \multicolumn{5}{|c|}{ Mean differences before and after treatment for both groups } \\
\hline & Before treatment & After treatment & $\begin{array}{l}t \text { - or } Z- \\
\text { values }\end{array}$ & \\
\hline \multicolumn{5}{|l|}{ Emotion Regulation Checklist } \\
\hline Emotion Regulation Subscale & $22.82(2.56)$ & $24.91(6.17)$ & $1.45+$ & \\
\hline Negativity/Lability Subscale & $38.00(5.33)$ & $33.73(5.00)$ & $2.03^{*}$ & \\
\hline \multicolumn{5}{|l|}{ Behavioral Monitoring Sheet } \\
\hline Frequency of episodes per hour & $.31(.16)$ & $.18(.09)$ & 1.18 & \\
\hline Duration in minutes per episode & $7.13(6.68)$ & $3.32(2.20)$ & $1.77+$ & \\
\hline \multicolumn{5}{|l|}{$\begin{array}{l}\text { Ben and the Bullies and James } \\
\text { and the Reading Group Vignettes }\end{array}$} \\
\hline Quantity scores & $1.36(.81)$ & $3.27(2.24)$ & $2.61^{*}$ & \\
\hline \multicolumn{5}{|l|}{ Self Confidence Rating Scale } \\
\hline Parental self-confidence - anger & $5.60(1.58)$ & $7.20(1.81)$ & $2.00^{*}$ & \\
\hline Parental self-confidence - anxiety & $4.73(1.90)$ & $7.36(1.12)$ & $2.82^{*}$ & \\
\hline Confidence in child - anger & $3.73(1.49)$ & $5.45(1.92)$ & $2.23^{*}$ & \\
\hline Confidence in child - anxiety & $2.82(1.25)$ & $5.55(1.81)$ & $2.82^{*}$ & \\
\hline
\end{tabular}

${ }^{*} p<.05,+p<.10$, one-tailed paired t-test or Wilcoxon signed rank test. 
may also be feasible and potentially helpful for teaching emotion regulation skills to younger children with ASD. Recommendations about the utility and efficacy of this developmentally modified CBT, however, await a full trial in future research.

\section{Acknowledgements}

The authors would like to thank the families for their participation and support in this study, the anonymous reviewers for their comments, and Drs Tony Attwood and Thomas Ollendick who served as consultants on this project. We also thank Akilah Paterson for her assistance in data coding, the staff at VT Autism Clinic and Fauquier Hospital, and group leaders Jessica Adams, Krystal Lewis, Alicia Lutman, Danielle Stover and Anthony Wells.

\section{References}

Attwood, T. (2004). Cognitive behaviour therapy for children and adults with Asperger's Syndrome. Behaviour Change, 21, 147-161. doi:10.1375/bech.21.3.147.55995.

Chalfant, A., Rapee, R. and Carroll, L. (2007). Treating anxiety disorders in children with high functioning autism spectrum disorders: a control trial. Journal of Autism and Developmental Disorders, 37, 1842-1857. doi:10.1007/s10803-006-0318-4.

de Bruin, E. I., Ferdinand, R. E., Meester, S., de Nijs, P. F. and Verheij, F. (2007). High rates of psychiatric co-morbidity in PDD-NOS. Journal of Autism and Developmental Disorders, 37, 877886. doi:10.1007/s10803-006-0215-x.

Sofronoff, K., Attwood, T. and Hinton, S. (2005). A randomized controlled trial of a cognitive behavioural intervention for anxiety in children with Asperger Syndrome. Journal of Child Psychology and Psychiatry, 46, 1152-1160. doi:10.1111/j.1469-7610.2005.00411.x.

Sofronoff, K., Attwood, T., Hinton, S. and Levin, I. (2007). A randomized controlled trial of a cognitive behavioural intervention for anger management in children diagnosed with Asperger Syndrome. Journal of Autism and Developmental Disorders, 37, 1203-1214. doi: 10.1007/s10803006-0262-3.

Wood, J., Drahota, A., Sze, K., Har, K., Chiu, A. and Langer, D. (2009). Cognitive behavioral therapy for anxiety in children with autism spectrum disorders: a randomized, controlled trial. Journal of Child Psychology and Psychiatry, 50, 224-234. doi:10.1111/j.14697610.2008.01948.x. 